\title{
INTERVENTION OF PHARMACOGENETIC PROFILING IN THERAPEUTICS OF KASHMIRI POPULATION, A LESSON LEARNED FROM CYP3A5*3 VARIANT
}

\author{
${ }^{1}$ Ashaqullah Bhat, ${ }^{2}$ Aadil Wani, ${ }^{1}$ Rajinder Kaur, ${ }^{2}$ Gulzar Ahmad Bhat, \\ ${ }^{2}$ Muzamil Ashraf Makhdoomi, ${ }^{2}$ Arif Bashir, ${ }^{2}$ Nida Sadiq, ${ }^{2}$ Muzamil Ali, \\ ${ }^{3}$ Showkat Ahmad Kadla and ${ }^{4}$ Bashir Ahmad Ganai \\ ${ }^{1}$ Department of Human Genetics, Punjabi University Patiala India, India \\ ${ }^{2}$ Departmet of Biochemistry, University of Kashmir, India \\ ${ }^{3}$ Department of Medicine, GMC Srinagar, India \\ ${ }^{4}$ Center of Research for Development, University of Kashmir, India
}

Received 2014-02-12; Revised 2014-07-08; Accepted 2014-07-11

\begin{abstract}
Human Cytochrome $\mathrm{P}_{450} 3 \mathrm{~A}$, a variant of Cytochrome P-450-dependent monooxygenases is a major drug metabolizing enzyme. CYP3A has evolved to catalyze the biotransformation of its substrate. A great deal of inter-individual variation in expression levels exists for CYP3A5 gene. The common polymorphism in CYP3A5 that induces a cryptic splice site has been shown to cause a significant reduction in its expression. Variation in CYP3A enzyme activity contributes greatly to variation in systemic clearance of CYP3A substrates and its oral bioavailability. The present study was designed to study the CYP3A $5 * 3$ polymorphism in the Kashmiri population. CYP3A $5 * 3$ genotypic status of 400 unrelated individuals of the Kashmiri population wasevaluated by PCR-RFLP method. The frequency of CYP3A5 variant in Kashmiri population was found similar to Caucasian but higher than that in Chinese and Japanese population. Our finding suggest that approximately $89.5 \%$ of study population carry CYP $3 \mathrm{~A} 5 * 3$ genotype and thus might be at an increased risk. So, detection of CYP3A5 variant alleles and knowledge about their allelic frequency in ethnic Kashmiris might play a key player in improved therapeutic intervention in Kashmiri population.
\end{abstract}

Keywords: Cytochrome $\mathrm{P}_{450}$, CYP3A5*3, Cyclosporina, PCR-RFLP

\section{INTRODUCTION}

Carcinogenesis is a multi factorial disease with genetic aberrations being at the heart of this dreadful disease. Chemical carcinogenesis has been earmarked as one of the main contributor to cause genetic instability. Most of the toxic substances are essentially procarcinogens and therefore require metabolic conversion to form highly reactive carcinogens. In humans, xenobiotic metabolizing enzymes which comprises of phase I and II enzymes, play a central role in the detoxification and eventually in systemic elimination of various toxic substances from the body. Human CYP3A super family of the phase I enzymes is chiefly responsible for the elimination of various endogenous as well as exogenous compounds (Eichelbaum et al., 2006). CYP3A subfamily of CytchromeP $_{450}$ consists of CYP3A4, CYP3A5, CYP3A7 and CYP3A43 are chiefly expressed in human liver and intestines. Among these, CYP3A4 and CYP3A5 are active forms of CYP3A in human liver and intestine (Gonzalez et al., 1988; Aoyama et al.,

\section{Corresponding Author: Bashir Ahmad Ganai, Department of Human Genetics, Punjabi University Patiala India}


1989; Komori et al., 1989; Domanski et al., 2001). Several studies have intimated that $30-85 \%$ of the inter-individual difference have in constancy in CYP3A prevalence due to genetic factors, such as Single Nucleotide Polymorphisms (SNPs) (Thummel and Wilkinson, 1998; Evans and Relling, 1999; Ozdemir et al., 2000). CYP regulated drug metabolism is prone to genetic in constancy that can ensue in enzyme's normal, low or no activity. The result of differences in CYP3A activity may be a clinical significance with respect to either efficacy of drug or biotransformation of drug-drug interactions as well as efficacious toxic substrates.

$\mathrm{CYP} 3 \mathrm{~A} 5 * 3(6986 \mathrm{~A} \rightarrow \mathrm{G})$ mutant allele is the prime defective allele among known alleles so far investigated in the $3^{\text {rd }}$ intone of CYP3A5 (Kuehl et al., 2001). Results have also revealed that the mutant allele lead to production of variant mRNA by alternative splicing which translates in the synthesis of truncated protein, resulting in the absence of CYP3A5 from some individuals. CYP3A5 may appear to contribute up to $50 \%$ of total hepatic CYP3Aprotein in individual carrying at least one allele of CYP3A $5 * 1$.

Thus, CYP3A5*3 mutant allele may play a prime role in inter-individual and inter-ethnic differences in the metabolic profile of many drugs used in therapeutics (Shin and Huang, 2002; King et al., 2003). Because evaluating the frequency ofCYP3A $5 * 3$ allele in a large scale Kashmiri population is likely to be instrumental in pharmacogeneticsresearch. Therefore, the present study was designed to evaluate the allelic frequency of CYP3A $5 * 3$ among a representative 400 Kashmiri individuals.

\section{MATERIALS AND METHODS}

\subsection{Subject Recruitment}

This hospital based cross sectional study was conducted over a period of fourteen months starting from May, 2012 to September, 2013, following approval by the ethical committee of Government Medical College (GMC), Srinagar, India. Informed consent was obtained from all the subjects.

Briefly, the patients recruited in the study were those who didn't have any previous cancer history and visited the hospital for some minor disease like hernia surgery, intestinal infection, skin burn, gastritis. The volunteer group consisting of 400 individuals (207 male and 193 female), aged between 35-60 years were recruited from the Department of Medicine, GMC Srinagar.

\subsection{Genomic DNA Isolation}

Five $\mathrm{ml}$ of venous blood was collected from each subject in a sterile EDTA coated vials and were subsequently stored at $-80^{\circ} \mathrm{C}$ for future use. Genomic DNA was isolated from the blood samples following Phenol-Chloroform method (Blin and Stafford, 1976). Integrity of the isolated genomic DNA was checked by running $5 \mu \mathrm{L}$ of the sample on $1 \%$ agarose gel.

\subsection{Genotyping of CYP $3 A 5 * 3$}

CYP3A5*3 polymorphism in intron $3^{\text {rd }}$ was analyzed through PCR-RFLP (restriction fragment length polymorphism) method.

PCR reactions contained $100 \mathrm{ng}$ of DNA, a 0.25 mM dNTPs (Sigma-Aldrich), 1× PCR buffer, 2.5 units of Taq polymerase (Ferment's) and $15 \mathrm{pmol}$ of forward 5/-CATGACTTAGTAGACAGATGAC-3/and reverse 5/-GGTCCAAACAGGGAAGAAATA$3 /$ Primers in a total volume of $25 \mu \mathrm{L}$. PCR cycling conditions included a 5-min initial denaturation step at $95^{\circ} \mathrm{C}$ followed by 35 cycles of the following: $50 \mathrm{~s}$ at $95^{\circ} \mathrm{C}, 50 \mathrm{~s}$ at an appropriate annealing temperature, 50 $\mathrm{s}$ at $72^{\circ} \mathrm{C}$ and a final extension step at $72^{\circ} \mathrm{C}$ for $5 \mathrm{~min}$. Quality of amp icons was assessed by running it in $2 \%$ agarose gel.

The PCR products so obtained were subjected to restriction digestion using SspI enzyme (Ferment as). The digested products of each subject were genotyped by running on $3 \%$ agarose gel (Fig. 1). CYP3A $5 * 3$ wild type allele possess the restriction site and thus produces three fragments of size 148, 125 and $20 \mathrm{bp}$ whereas mutant allele lacks restriction site and on digestion produces two fragments of size 168 and $125 \mathrm{bp}$.

\subsection{Statistical Analysis}

All the statistical analyses were performed with Statistical Package for the Social Science (SPSS version 15.0.). Chi square test was put in use for comparison of the allele and genotypic frequency variations between different populations. The divergences from the Hardy-Weinberg equilibrium for allele and genotype frequencies for the various SNPs were assessed by Fisher's exact test. The 95\% confidence intervals were enumerated for all observed allele frequencies. A $\mathrm{p}<0.05$ was considered statistically

significant. 


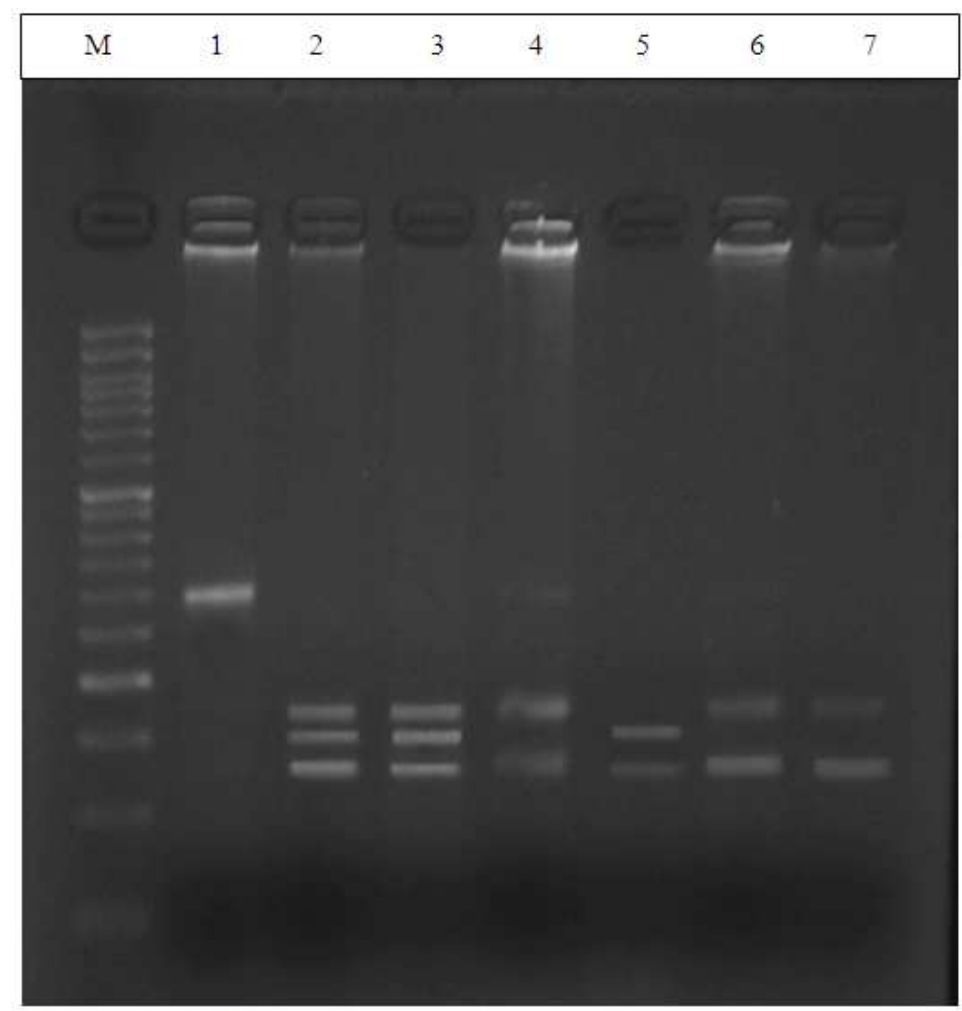

M-50 bp DNA ladder

Lane 1-Untreated PCR amplicon of $293 \mathrm{bp}$

Lane 2, 3-Heterozygous genotype

Lane 5-Homozygous wild genotype

Lane 4,6 and 7-Homozygous mutant genotype

Fig. 1. PCR-Restriction Fragment Length Polymorphism analysis (PCR-RFLP) of CYP3A5*3 polymorphism

\section{RESULTS}

The distribution of genotypic frequencies of CYP3A $5 * 3$ of the present cohort of four hundred volunteer patients is summarized in Table 1. The genotypic frequency observed for homozygous wild type $(1 * / 1 *)$ was $3 \%(12 / 400)$. The genotypic frequencies analyzed for heterozygous genotype $(1 * / 3 *)$ and homozygous mutant type $(3 * / 3 *)$ were $7.5 \%(30 / 400)$ and $89.5 \%(358 / 400)$ respectively (Table 1). The genotypic distribution of CYP3A $5 * 3 \mathrm{SNP}$ at 3rd intone position is in Hardy-Weinberg equilibrium in the present study.

The frequency of wild allele in the patients under study was $0.067(54 / 800)$. The mutant allele frequency was $0.93(746 / 800)$. Therefore, the percentage calculated for wild and mutant allele of CYP3A $5 * 3$ in the present patients was 6.75 and 93.25 respectively.

Comparison of the allelic and genotypic frequency of the present cohort of the Kashmiri population with other ethnic populations was done. We observed that the allelic frequency of $\mathrm{CYP} 3 \mathrm{~A} 5 * 3$ in the Kashmiri population was found to be $93 \%$, which is similar to Dutch Caucasian $(91.7 \%) \mathrm{p}=0.80, \chi^{2}=0.06$, but significantly higher than in Chinese $(77.8 \%) \mathrm{p}=0.02, \chi^{2}$ $=5.14$, Japanese population $(76.8 \%) \mathrm{p}=0.03 \chi^{2}=4.55$ and African Americans (47.5\%) $\mathrm{p}=0.01 \chi^{2}=5.87$. Also, CYP3A5*3 heterozygous and homozygous mutant genotype was found to be similar in Dutch Caucasian, but was higher as compared to the Chinese, Japanese and African American (Table 2). 
Ashaqullah Bhat et al. / OnLine Journal of Biological Sciences 14 (3): 170-174, 2014

Table 1. Allelic and genotypic Frequency of CYP3A5 variant alleles in the Kashmiri population $(\mathrm{n}=400)$

\begin{tabular}{llllll}
\hline Polymorphism & Effect & Allele & Frequency & Genotypic & Frequency \\
\hline CYP3A5*3 & $1^{*}$ & $3^{*}$ & $1 * / 1^{*}$ & $1 * / 3^{*}$ & $3^{* / 3 *}$ \\
Splicing defect & 0.067 & 0.933 & 3 & 7.5 & 89.5 \\
\hline
\end{tabular}

Table 2. Allelic and genotype frequency of the CYP3A5 in Kashmiri population and other ethnic population

\begin{tabular}{|c|c|c|c|c|c|c|}
\hline \multirow[b]{2}{*}{ Population } & \multirow{2}{*}{$\begin{array}{l}\mathrm{Na} \\
-1 * \\
\end{array}$} & \multicolumn{2}{|c|}{ Allelic frequency } & \multicolumn{3}{|c|}{ Genotype frequency (\%) } \\
\hline & & $3 *$ & $1 * / 1 *$ & $1 * / 3 *$ & $3 * / 3 *$ & References \\
\hline Kashmiri population & 800 & 0.067 & 0.900 & 37.5 & 89.5 & in this study \\
\hline Chinese & 604 & 0.222 & 0.778 & 7.3 & 29.8 & 62.9 (Yong-Fang et al., 2005) \\
\hline Japanese & 400 & 0.233 & 0.768 & 7.0 & 32.5 & 60.5 (Kuehl et al., 2001) \\
\hline Dutch Caucasian & 1000 & 0.083 & 0.917 & 0.2 & 16.7 & 83.1 (Komori et al., 1989) \\
\hline African American & 40 & 0.450 & 0.475 & 25.0 & 25.0 & 35.0 (First et al., 1996) \\
\hline
\end{tabular}

\section{DISCUSSION}

Despite the marked advances in pharmacokinetics and drug therapy, fractions of patients don't respond favorably or experiences severe adverse drug side effects. Pharmacogenetic studies have revealed the essence of polymorphism in drug metabolizing enzymes, transporters and receptors which contribute to variable drug response as per the individual's genetic makeup (Eichelbaum et al., 2006). CYP3A5 is forms are responsible for metabolizing about $50 \%$ of all clinically approved drugs (Arvanitidas et al., 2007). Thus polymorphism of CYP family may have most impact on the fate of these and other therapeutic drugs whose metabolism they regulate. Therefore, the present study was designed to evaluate the genetic status of the CYP3A5 gene in the large ethnic Kashmiri population.

Our results showing high prevalence $(89.5 \%)$ of the defective CYP3A5*3 allele in Kashmiri population is in agreement with the other studies conducted in India and elsewhere (Bajpai et al., 2010; Fukuen et al., 2002). The present study reveals a marked interethnic variation in CYP3A5 allelic and genotypic frequencies between Kashmiri population and other ethnic populations like African Americans, Dutch Caucasians and Chinese. The CYP3A $5 * 3$ variant allele and homozygote are more frequent in Kashmiri population and Dutch Caucasians than in Chinese and is much less common in African Americans (Ozdemir et al., 2000; Van Schaik et al., 2002). Interethnic variations in polymorphism in drug metabolizing enzymes like CYP3A5 may contribute to drug efficiency and its toxicity. For instance, Cyclosporine A (CsA), an immunosuppressive agent widely administrated in the patients who receive organ transplants which inhibits the activation of naïve $\mathrm{T}$ cells is metabolized by CYP3A4 andCYP3A5. It has been reported that the bioavailability of CsA was found significantly lower in African-American patients with renal transplantation than those in Caucasian-American patients, in contrast to dosing requirement of CsA, African-American patients had significantly higher dosing requirement compared with Caucasian and American patients, when the same therapeutic regimen was maintained (First et al., 1996). This may be due to the frequency of the CYP3A $5 * 3$ mutant allele is higher in Caucasian-American than AfricanAmerican. So in the wake of above evidences, our study may represent the basis for the intervention of pharmacogenetic profiling in therapeutic options in Kashmiri population.

\section{CONCLUSION}

The present study shows that genotypic screening for CYP3A5*3 alleles in the ethnic Kashmiri population is highly relevant. Thus population based screening for CYP3A5*3mutant allele will help understand the disease risk as well as pharmacokinetics of many drugs.

\section{ACKNOWLEDGEMENT}

The study was supported by a grant from the $\mathrm{j}$ and $\mathrm{k}$ State Council for Science and Technology. We thank all the consultants and the other paramedical staff of the Department of Hematology at GMC, Srinagar for their invaluable help during sample collection. We appreciate the participation of all the subjects who volunteered for this study. The authors declare that they have no conflict of interest. 


\section{REFERENCES}

Aoyama, T., S. Yamano, D.J. Waxman, D.P. Lapenson and U.A. Meyer et al., 1989. Cytochrome P-450 hPCN3, a novel cytochrome P-450 IIIA gene product that is differentially expressed in adult human liver. cDNA and deduced amino acid sequence and distinct specificities of cDNA-expressed hPCN1 and hPCN3 for the metabolism of steroid hormo. J. Biochem., 264: 10388-95. PMID: 2732228

Arvanitidas, K., G. Ragia, M. Iordanidous, S. Kyriaki and A. Xanthi et al., 2007. Genetic polymorphisms of drug-metabolizing enzymes CYP2D6, CYP2C9, CYP2C19 and CYP3A5 in the Greek population. Fundamental ClinPharmacol, 21: 419-26.

Bajpai, P., A.K. Tripathi and D. Agrawal, 2010. Genetic polymorphism of CYP3A5 in Indian chronic myeloid leukemia patients. Mol. Cell Biochem., 336: 49-54. PMID: 19823770

Blin, N. and D.W. Stafford, 1976. A general method for isolation of high molecular weight DNA from eukaryotes. Nucleic Acids Res., 3: 2303-8. DOI: 10.1093/nar/3.9.2303

Domanski, T.L., C. Finta, J.R. Halpert and P.G. Zaphiropoulos, 2001. cDNA cloning and initial characterization of CYP3A43, a novel human Cytochrome P450. Mol. Pharmacol., 59: 386-92.

Eichelbaum, M., M. Ingelman-Sundberg, W.E. Evans, 2006. Pharmacogenomics and individualized drug therapy. Ann. Rev. Med., 57: 119-37. PMID: 16409140

Evans, W.E. and M.V. Relling, 1999. Pharmacogenetics: Translating functional genomics into rational therapeutics. Science, 286: 487-91. DOI: 10.1126/science.286.5439.487

First, M.R., T.J. Schroeder, A.P. Monaco, M.A. Simpson and J.J. Curtis et al., 1996. Cyclosporine bioavailability: Dosing implications and impact on clinical outcomes in select transplantation subpopulation. Clin. Transplant, 10: 55-59. PMID: 8652899

Fukuen, S., T. Fukuda, H. Maune, Y. Ikenaga and I. Yamamoto et al., 2002. Novel detection assay by PCR-RFLP and frequency of the CYP3A5 SNPs, CYP3A5*3 and $* 6$, in a Japanese population. Pharmacogenetic, 12: 331-4. PMID: 12042671
Gonzalez, F.J., B.J. Schmid, M. Umeno, O.W. Mcbride and J.P. Hardwick et al., 1988. Human P450PCN1: Sequence, chromosome localization and direct evidence through cDNA expression that P450PCN1 is nifedipine oxidase. DNA, 7: 79-86. DOI: 10.1089/dna.1988.7.79

King, B.P., J.B. Leathart, E. Mutch, F.M. Williams, A.K. Daly, 2003. CYP3A5 phenotype-genotype correlations in a British population. Br. J. Clin. Pharmacol., 55: 625-9. DOI: 10.1046/j.13652125.2003.01798.x

Komori, M., K. Nishio, H. Ohi, M. Kitada, T. Kamataki, 1989. Molecular cloning and sequence analysis of cDNA containing the entire coding region for human fetal liver cytochrome P-450. J. Biochem., Tokyo, 105:161-3. PMID: 2722762

Kuehl, P., J. Zhang, Y. Lin, J. Lamba and M. Assem et al., 2001. Sequence diversity in CYP3A promoters and characterization of the genetic basis of polymorphic CYP3A5 expression. Nat. Genet., 27: 383-91. DOI: $10.1038 / 86882$

Ozdemir, V., W. Kalowa B.K. Tang, A.D. Paterson and S.E. Walker et al., 2000. Evaluation of the genetic component of variability in CYP3A4 activity: A repeated drug administration method. Pharmacogenetics, 10: 373-88. DOI: 10.1097/00008571-200007000-00001

Shin, P.S. and J.D. Huang, 2002. Pharmacokinetics of midazolam and 1'-hydroxymidazolam in Chinese with different CYP3A5 genotypes. Drug Metab. Disposit., 30: 1491-1496. DOI: 10.1124/dmd.30.12.1491

Thummel, K.E. and G.R. Wilkinson, 1998. Invitro and invivo drug interactions involving human CYP3A. Ann. Rev. Pharmacol Toxicol, 38: 389-430. DOI: 10.1146/annurev.pharmtox.38.1.389

Van Schaik, R.H., I.P. Van Der Heiden, J.N. Van Den Anker and J. Lindemans, 2002. CYP3A5 variant allele frequencies in Dutch Caucasians. Clin Chem., 48: 1668-1671. PMID: 12324482

Yong-Fang, H., J. He, G.L. Chen, D. Wang and H.H. Zhou et al., 2005. CYP3A5*3 and CYP3A4*18 single nucleotide polymorphisms in a Chinese population. Clin. Chimica Acta, 353: 187-192. DOI: 10.1016/j.ccen.2004.11.005 